\title{
Action Recognition by Hierarchical Sequence Summarization
}

\author{
Yale Song $^{1}$, Louis-Philippe Morency ${ }^{2}$, Randall Davis ${ }^{1}$ \\ ${ }^{1}$ MIT Computer Science and Artificial Intelligence Laboratory \\ ${ }^{2}$ USC Institute for Creative Technology \\ \{yalesong, davis\}@csail.mit.edu, morency@ict.usc.edu
}

\begin{abstract}
Recent progress has shown that learning from hierarchical feature representations leads to improvements in various computer vision tasks. Motivated by the observation that human activity data contains information at various temporal resolutions, we present a hierarchical sequence summarization approach for action recognition that learns multiple layers of discriminative feature representations at different temporal granularities. We build up a hierarchy dynamically and recursively by alternating sequence learning and sequence summarization. For sequence learning we use CRFs with latent variables to learn hidden spatiotemporal dynamics; for sequence summarization we group observations that have similar semantic meaning in the latent space. For each layer we learn an abstract feature representation through non-linear gate functions. This procedure is repeated to obtain a hierarchical sequence summary representation. We develop an efficient learning method to train our model and show that its complexity grows sublinearly with the size of the hierarchy. Experimental results show the effectiveness of our approach, achieving the best published results on the ArmGesture and Canal9 datasets.
\end{abstract}

\section{Introduction}

Recent progress has shown that learning from hierarchical feature representations leads to significant improvements in various computer vision tasks, including spatial pyramids of image patches in object detection [11], higher order potentials in object segmentation [7], and the deep learning with multiple hidden layers [12, 17]. Although there is much difference in algorithmic details, these approaches share the common goal of learning from hierarchical feature representations in order to capture high-level concepts that are otherwise difficult to express with a single representation approach.

*This work was supported in part by ONR \#N000140910625, by NSF IIS-1018055, and by the U.S. Army RDECOM.

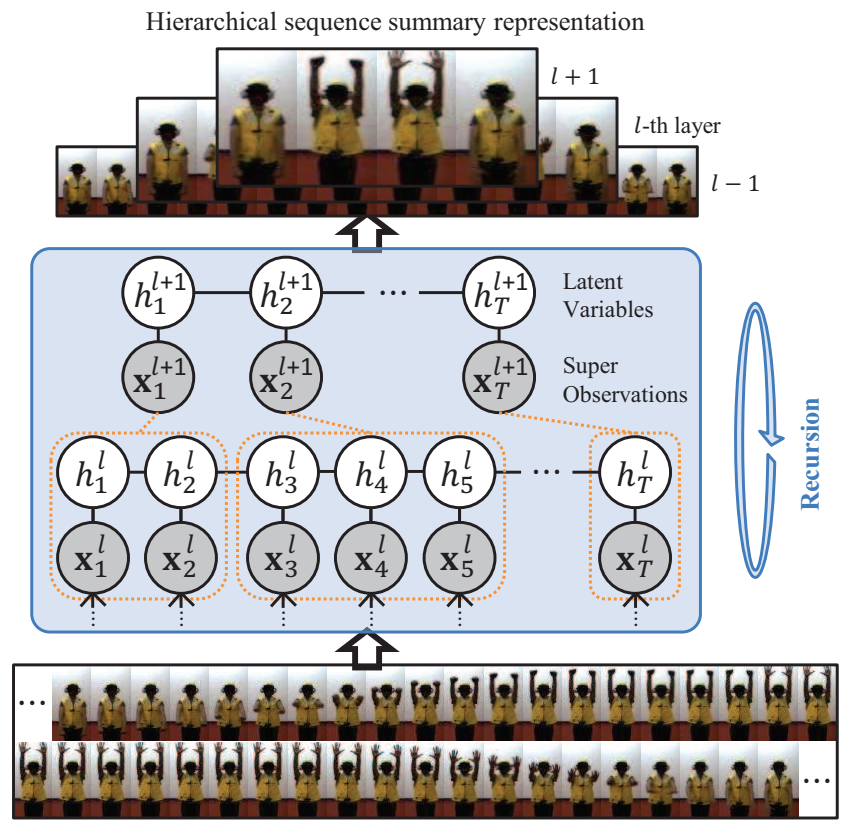

Figure 1. Human activity data contains information at various temporal resolutions, having many similar observations with occasional and irregular changes. This makes it difficult to capture discriminative information from a single temporal resolution. We build a hierarchical representation of sequence and learn from multiple layers of different feature representations.

Action recognition is one particular area that can benefit from such representations, because human activity data contains information at various spatio-temporal resolutions. People may, for example, perform gestures slowly to emphasize a point, but more rapidly on unintentional movements or meaningless gestures. The resulting data stream will have many similar observations with occasional and irregular changes. As a result, capturing discriminative information from a single temporal representation may prove to be difficult.

The $\mathrm{C}++$ implementation of our model is available at http:// people.csail.mit.edu/yalesong/ 
Numerous approaches have been proposed to learn from a hierarchical representation of human action [13, 23, 8, 26, 12]. Following the popular bag-of-words approach [9, 10], several efforts have proposed to construct a hierarchical representation of local feature descriptors [23, 8, 26]; although shown to be effective, these approaches used learning algorithms that ignore the temporal order of sequential data (such as SVM and MKL), which limits their application to many real-world problems that exhibit temporal structures [16, 24]. Other efforts have proposed sequence models to learn hierarchical feature representation [13, 28, 12, 6, 24]. Notably, Le et al. [12] showed that learning a hierarchical feature representation leads to significant improvements in action recognition. The challenge here is efficiency: for deep belief networks [5] solving the optimization problems when the size of the hierarchy is large remains a challenge [18].

This paper presents a hierarchical sequence summarization approach for action recognition that learns multiple layers of discriminative feature representations at different temporal granularities. Our approach is motivated by the observation that human activity data contains information at various temporal resolutions. We build up a hierarchical representation dynamically and recursively by alternating sequence learning and sequence summarization. For sequence learning we use CRFs with latent variables [16], but modify the standard feature function to use a set of nonlinear gate functions, as used in neural networks, to automatically learn a discriminative feature representation. For sequence summarization we group observations that have similar semantic meaning in the latent space, defining a similarity metric using the posteriors of latent variables, and using an efficient graph-based variable grouping algorithm [3] to obtain a sequence summary representation. As the hierarchy builds, we learn discriminative feature representations that contain ever more high-level spatio-temporal information. We have developed an efficient optimization method to train our model; its complexity grows only sublinearly as the size of the hierarchy grows.

Section 2 reviews some of the related work, Section 3 details our Hierarchical Sequence Summarization (HSS) model, and Section 4 reports experiments using three human activity datasets with various tasks. We conclude in Section 5 with contributions and future directions.

\section{Related Work}

Learning from a hierarchical feature representation has been a recurring theme in action recognition $[13,23,8,26$, 12]. One approach uses the popular bag-of-words approach, which detects spatio-temporal interest points (STIP) [9] at local video volumes, constructs a bag-of-words representation of HOG/HOF features extracted around STIPs, and learns an SVM classifier to categorize actions [10]. This has been used to construct a hierarchical feature representation that is more discriminative and context-rich $[23,26,8]$. Sun et al. [23] defined three levels of context hierarchy with SIFT-based trajectories, while Wang et al. [26] learned interactions within local contexts at multiple spatio-temporal scales. Kovashaka and Grauman [8] proposed to learn class conditional visual words by grouping local features of motion and appearance at multiple space-time scales. While these approaches showed significant improvements over the local feature representation, they use non-temporal machine learning algorithms to classify actions (e.g., SVM and MKL), limiting their application to real-world scenarios that exhibit complex temporal structures [16, 24].

Sequence learning has been a well-studied topic in machine learning (e.g., HMM and CRF), and has been used successfully in action recognition [16, 27, 24]. Quattoni et al. [16] incorporated latent variables into CRF (HCRF) to learn hidden spatio-temporal dynamics, while Wang et al. [27] applied the max-margin learning criterion to train HCRFs. While simple and computationally efficient, the performance of HCRFs has been shown to decrease when the data has complex input-output relationships [15, 28]. To overcome this limitation, Peng et al. [15] presented Conditional Neural Fields (CNF) that used gate functions to extract nonlinear features representations. However, these approaches are defined over a single representation and thus cannot benefit from the additional information that hierarchical representation provides.

Our model has many similarities to the deep learning paradigm [1], such as learning from multiple hidden layers with non-linear operations. Deep belief networks (DBN) [5] have been shown to outperform other "shallow" models in tasks such as digit recognition [5], object recognition [18], and face recognition [6]. Recently, Le et al. [12] applied an extension of Independent Subspace Analysis with DBN to action recognition. However, obtaining an efficient learning algorithm that is scalable with the number of layers still remains a challenge [5, 18]. Compared to DBN, the learning complexity of our method grows sublinearly with the size of the hierarchy.

Previous approaches to learning with multiple representations using HCRF (e.g., [28]) define each layer as a combination of the original observation and the preceding layer's posteriors, at the same temporal resolution. Our work learns each layer at temporally coarser-grained resolutions, making our model capable of learning ever-more high-level concepts that incorporate the surrounding context (e.g., what comes before/after).

\section{Hierarchical Sequence Summarization}

We propose to capture complex spatio-temporal dynamics in human activity data by learning from a hierarchical sequence summary representation. Intuitively, each layer in 
the hierarchy is a temporally coarser-grained summary of the sequence from the preceding layer, and is built dynamically and recursively by grouping observations that have similar semantic meaning in the latent space.

Our approach builds the hierarchy by alternating sequence learning and sequence summarization. We define our notation in Section 3.1, describe sequence learning in Section 3.2 and sequence summarization in Section 3.3. We then formally define our model in Section 3.4 and explain an efficient optimization procedure in Section 3.5.

\subsection{Notation}

Input to our model is a time-ordered sequence $\mathrm{x}=$ $\left[\mathbf{x}_{1} ; \cdots ; \mathbf{x}_{T}\right]$ of length $T$ (the length can vary across sequences); each per-frame observation $\mathbf{x}_{t} \in \mathbb{R}^{D}$ is of dimension $D$ and can be any type of action feature (e.g., body pose configuration [16], bag-of-words representation of $\mathrm{HOG} / \mathrm{HOF}$ [10], etc.). Each sequence is labeled $y$ from a finite alphabet set, $y \in \mathcal{Y}$.

We denote a sequence summary at the $l$-th layer in the hierarchy by $\mathbf{x}^{l}=\left[\mathbf{x}_{1}^{l} ; \cdots ; \mathbf{x}_{T}^{l}\right]$. A super observation $\mathbf{x}_{t}^{l}$ is a group of observations from the preceding layer, and we define $c\left(\mathbf{x}_{t}^{l}\right)$ as a reference operator of $\mathbf{x}_{t}^{l}$ that returns the group of observations; for $l=1$ we set $c\left(\mathbf{x}_{t}^{l}\right)=\mathbf{x}_{t}$.

Because our model is defined recursively, most procedures at each layer can be formulated without specifying the layer index. In what follows, we omit $l$ whenever it is clear from the context; we also omit it for the original sequence, i.e., the first layer $(l=1)$.

\subsection{Sequence Learning}

Following [16], we use CRFs with latent variables to capture hidden dynamics in each layer in the hierarchy. Using a set of latent variables $\mathbf{h} \in \mathcal{H}$, the conditional probability distribution is defined as

$$
p(y \mid \mathbf{x} ; \mathbf{w})=\frac{1}{Z(\mathbf{x} ; \mathbf{w})} \sum_{\mathbf{h}} \exp F(y, \mathbf{h}, \mathbf{x} ; \mathbf{w})
$$

where $\mathbf{w}$ is a model parameter vector, $F(\cdot)$ is a generic feature function, and $Z(\mathbf{x} ; \mathbf{w})=\sum_{y^{\prime}, \mathbf{h}} \exp F\left(y^{\prime}, \mathbf{h}, \mathbf{x} ; \mathbf{w}\right)$ is a normalization term.

Feature Function: We define the feature function as

$$
\begin{aligned}
F(y, \mathbf{h}, \mathbf{x} ; \mathbf{w}) & =\sum_{t} f^{1}(\mathbf{h}, \mathbf{x}, t ; \mathbf{w})+\sum_{t} f^{2}(y, \mathbf{h}, t ; \mathbf{w}) \\
& +\sum_{t} f^{3}(y, \mathbf{h}, t, t+1 ; \mathbf{w})
\end{aligned}
$$

Our definition of feature function is different from that of [16] to accommodate the hierarchical nature of our approach. Specifically, we define the super observation feature function that is different from [16].
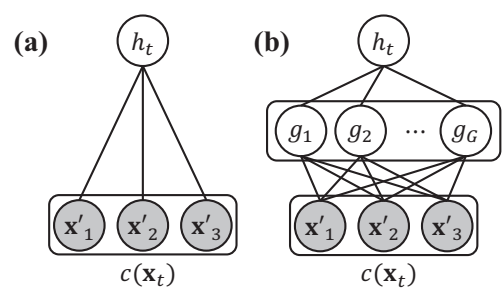

Latent

variable

Gate

functions

Super

observations

Figure 2. Illustration of our super observation feature function. (a) Observation feature function similar to Quattoni et al. [16], (b) our approach uses an additional set of gate functions to learn an abstract feature representation of super observations.

Let $\mathbb{1}[\cdot]$ be an indicator function, and $y^{\prime} \in \mathcal{Y}$ and $\left(h^{\prime}, h^{\prime \prime}\right) \in \mathcal{H}$ be the assignments to the label and latent variables, respectively. The second and the third terms in Equation 2 are the same as those defined in [16], i.e., the label feature function $f^{2}(\cdot)=w_{y, h} \mathbb{1}\left[y=y^{\prime}\right] \mathbb{1}\left[h_{t}=h^{\prime}\right]$ and the transition feature function $f^{3}(\cdot)=w_{y, h, h} \mathbb{1}[y=$ $\left.y^{\prime}\right] \mathbb{1}\left[h_{t}=h^{\prime}\right] \mathbb{1}\left[h_{t+1}=h^{\prime \prime}\right]$.

Our super observation feature function (the first term of Equation 2) incorporates a set of non-linear gate functions $G$, as used in neural networks, to learn an abstract feature representation of super observations (see Figure 2 (b)). Let $\psi_{g}(\mathbf{x}, t ; \mathbf{w})$ be a function that computes, using a gate function $g(\cdot)$, an average of gated output values from each observation contained in a super observation $\mathbf{x}^{\prime} \in c\left(\mathbf{x}_{t}\right)$,

$$
\psi_{g}(\mathbf{x}, t ; \mathbf{w})=\frac{1}{\left|c\left(\mathbf{x}_{t}\right)\right|} \sum_{\mathbf{x}^{\prime} \in c\left(\mathbf{x}_{t}\right)} g\left(\sum_{d} w_{g, d} x_{d}^{\prime}\right)
$$

We adopt the popular logistic function as our gate function, $g(z)=1 /(1+\exp (-z))$, which has been shown to perform well in various tasks [1]. We define our super observation feature function as

$$
f^{1}(\mathbf{h}, \mathbf{x}, t ; \mathbf{w})=\sum_{g \in G} w_{g, h} \mathbb{1}\left[h_{t}=h^{\prime}\right] \psi_{g}(\mathbf{x}, t ; \mathbf{w}) .
$$

where each $g \in G$ has the same form. The set of gate functions $G$ creates an additional layer between latent variables and observations, and has a similar effect to that of the neural network. That is, this feature function automatically learns an abstract representation of super observations, and thus provides more discriminative information for capturing complex spatio-temporal patterns in human activity data.

To see the effectiveness of the gate functions, consider another definition of the observation feature function, one without the gate functions (see Figure 2 (a)),

$$
f^{1}(\mathbf{h}, \mathbf{x}, t ; \mathbf{w})=\frac{1}{\left|c\left(\mathbf{x}_{t}\right)\right|} \sum_{\mathbf{x}^{\prime}} \sum_{d} w_{h, d} \mathbb{1}\left[h_{t}=h^{\prime}\right] x_{d}^{\prime}
$$

This does not have the automatic feature learning step, and simply represents the feature as an average of the linear 


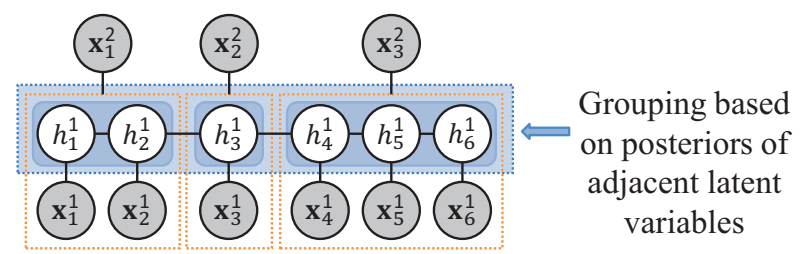

Figure 3. Illustration of sequence summarization. We generate a sequence summary by grouping neighboring observations that have similar semantic labeling in the latent space.

combinations of features $x_{d}^{\prime}$ and weights $w_{h, d}$. As evidenced by the deep learning literature [1, 12], and consistent with our experimental result in Section 4, the step of non-linear feature learning leads to a more discriminative representation.

Complexity Analysis: Our model parameter vector is $\mathbf{w}=\left[w_{g, h} ; w_{g, d} ; w_{y, h} ; w_{y, h, h}\right]$ and has the dimension of $G H+G D+Y H+Y H H$, with the number of gate functions $G$, the number of latent states $H$, the feature dimension $D$, and the number of class labels $Y$. Given a chain-structured sequence $\mathbf{x}$ of length $T$, we can solve the inference problem at $O\left(Y T H^{2}\right)$ using a belief propagation algorithm.

\subsection{Sequence Summarization}

There are many ways to summarize $\mathbf{x}^{l}$ to obtain a temporally coarser-grained sequence summary $\mathbf{x}^{l+1}$. One simple approach is to group observations from $\mathrm{x}^{l}$ at a fixed time interval, e.g., collapse every two consecutive observations and obtain a sequence with half the length of $\mathbf{x}^{l}$. However, as we show in our experiments, this approach may fail to preserve important local information and result in over-grouping and over-smoothing.

We therefore summarize $\mathrm{x}^{l}$ by grouping observations at an adaptive interval, based on how similar the semantic labeling of observations are in the latent space. We work in the latent space because it has learned to maximize class discrimination and thus provides more semantically meaningful information. Said slightly differently, the similarity of latent variables is a measure of the similarity of the corrsponding observations, but in a space more likely to discriminative appropriately.

Sequence summarization can be seen as a variable grouping problem with a piecewise connectivity constraint. We use the well-established graph-based variable grouping algorithm by Felzenszwalb et al. [3], with a modification on the similarity metric. The algorithm has the desirable property that it preserves detail in low-variance groups while ignoring detail in high-variance groups, producing a grouping of variables that is globally coherent. The pseudocode of the algorithm is given in Algorithm 1.

The Algorithm: Let $\mathcal{G}=(\mathcal{V}, \mathcal{E}, \mathcal{W})$ be a weighed graph at the $l$-th layer, where $\mathcal{V}$ is a set of nodes (latent variables),

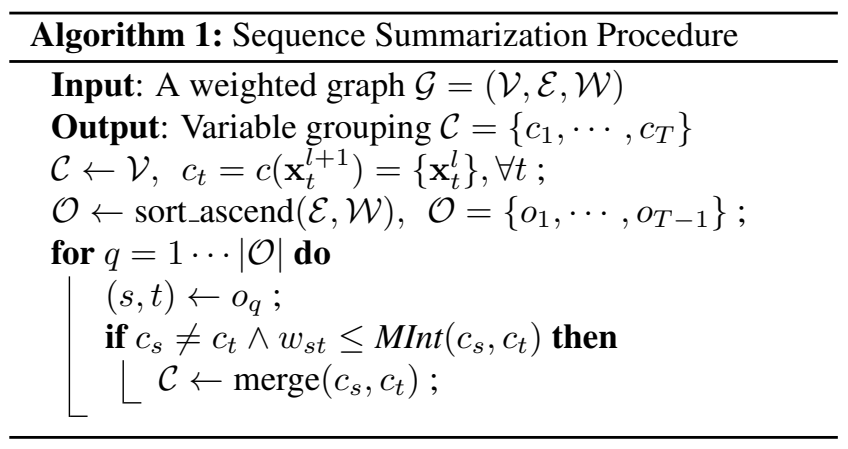

$\mathcal{E}$ is a set of edges induced by a linear chain, and $\mathcal{W}$ is a set of edge weights defined as the similarity between two nodes. The algorithm produces a set of super observations $\mathcal{C}=\left\{c\left(\mathbf{x}_{1}^{l+1}\right), \cdots, c\left(\mathbf{x}_{T}^{l+1}\right)\right\}$.

The algorithm merges $c\left(\mathbf{x}_{s}^{l+1}\right)$ and $c\left(\mathbf{x}_{t}^{l+1}\right)$ if the difference between the groups is smaller than the minimum internal difference within the groups. Let the internal difference of a group $c$ be $\operatorname{Int}(c)=\max _{(s, t) \in \operatorname{mst}\left(c, \mathcal{E}_{c}\right)} w_{s t}$, i.e., the largest weight in the minimum spanning tree of the group $c$ with the corresponding edge set $\mathcal{E}_{c}$. The minimum internal difference between two groups $c_{s}$ and $c_{t}$ is defined as $\operatorname{MInt}\left(c_{s}, c_{t}\right)=\min \left(\operatorname{Int}\left(c_{s}\right)+\tau\left(c_{s}\right), \operatorname{Int}\left(c_{t}\right)+\tau\left(c_{t}\right)\right)$ where $\tau\left(c_{s}\right)=\tau /\left|c_{s}\right|$ is a threshold function; it controls the degree to which the difference between two groups must be greater than their internal differences in order for there to be evidence of a boundary between them.

Similarity Metric: We define the similarity between two nodes (i.e., the weight $w_{s t}$ ) as

$$
w_{s t}=\sum_{y, h^{\prime}}\left|p\left(h_{s}=h^{\prime} \mid y, \mathbf{x} ; \mathbf{w}\right)-p\left(h_{t}=h^{\prime} \mid y, \mathbf{x} ; \mathbf{w}\right)\right|
$$

that is, it is the sum of absolute differences of the posterior probabilities between the two corresponding latent variables, marginalized over the class label. ${ }^{1}$

Complexity Analysis: As shown in [3], this sequence summarization algorithm runs quite efficiently in $O(T \log T)$ with the sequence length $T$.

\subsection{The HSS Model}

We formulate our model, Hierarchical Sequence Summarization (HSS), as the conditional probability distribution

$$
p(y \mid \mathbf{x} ; \mathbf{w}) \propto p\left(y \mid \mathbf{x}^{1}, \cdots, \mathbf{x}^{\mathcal{L}} ; \mathbf{w}\right) \propto \prod_{l=1}^{\mathcal{L}} p\left(y \mid \mathbf{x}^{l} ; \mathbf{w}^{l}\right)
$$

where $p\left(y \mid \mathbf{x}^{l} ; \mathbf{w}^{l}\right)$ is obtained using Equation 1. Note the layer-specific model parameter vector $\mathbf{w}^{l}, \mathbf{w}=$ $\left[\mathbf{w}^{1} ; \cdots ; \mathbf{w}^{\mathcal{L}}\right]$.

\footnotetext{
${ }^{1}$ Other metrics can also be defined in the latent space. We experimented with different weight functions, but the performance difference was not significant. We chose this definition because it performed well across different datasets and is computationally simple.
} 
The first derivation comes from our reformulation of $p(y \mid \mathbf{x} ; \mathbf{w})$ using hierarchical sequence summaries, the second comes from the way we construct the sequence summaries. To see this, recall that we obtain a sequence summary $\mathbf{x}^{l+1}$ given the posterior of latent variables $p\left(\mathbf{h}^{l} \mid y, \mathbf{x}^{l} ; \mathbf{w}^{l}\right)$, and the posterior is computed based on the parameter vector $\mathbf{w}^{l}$; this implies that $\mathbf{x}^{l+1}$ is conditionally independent of $\mathbf{x}^{l}$ given $\mathbf{w}^{l}$. To make our model tractable, we assume that a parameter vector at each layer $\mathbf{w}^{l}$ is independent of each other. As a result, we can express the second term as the product of $p\left(y \mid \mathbf{x}^{l} ; \mathbf{w}^{l}\right)$.

\subsection{Incremental Optimization}

Given $\mathcal{D}=\left\{\left(\mathbf{x}_{i}, y_{i}\right) \mid \mathbf{x}_{i} \in \mathbb{R}^{D \times T_{i}}, y_{i} \in \mathcal{Y}\right\}_{i=1}^{N}$ as a training dataset, the standard way to find the optimal solution $\mathrm{w}^{*}$ is to define an objective function as

$$
\min _{\mathbf{w}} L(\mathbf{w})=\mathcal{R}(\mathbf{w})-\sum_{i=1}^{N} \log p\left(y_{i} \mid \mathbf{x}_{i} ; \mathbf{w}\right)
$$

with a regularization term $\mathcal{R}(\mathbf{w})=\frac{1}{2 \sigma^{2}}\|\mathbf{w}\|^{2}$, i.e., the $\log$ of a Gaussian prior with variance $\sigma^{2}, p(\mathbf{w}) \sim$ $\exp \left(\frac{1}{2 \sigma^{2}}\|\mathbf{w}\|^{2}\right)$, then solve it using gradient descent [14].

Unfortunately, because of the hierarchical nature of our approach, the objective function needs to be changed. In our approach only the original sequence $\mathrm{x}^{1}$ is available at the outset; to generate a sequence summary $\mathbf{x}^{l+1}$ we need the posterior $p\left(\mathbf{h}^{l} \mid y, \mathbf{x}^{l} ; \mathbf{w}^{l}\right)$, and the quality of the posterior relies on an estimate of the solution $\mathbf{w}^{l}$ obtained so far.

We therefore perform incremental optimization [4], where, at each layer $l$, we solve for only the necessary part of the solution while fixing all the others, and iterate the optimization process, incrementing $l$. At each layer $l$ of the incremental optimization, we solve

$$
\min _{\mathbf{w}^{l}} L\left(\mathbf{w}^{l}\right)=\mathcal{R}\left(\mathbf{w}^{l}\right)-\sum_{i=1}^{N} \log p\left(y_{i} \mid \mathbf{x}_{i}^{l} ; \mathbf{w}^{l}\right)
$$

This layer-specific optimization problems is solved using gradient descent with a standard quasi-newton method, LBFGS [14], chosen because of its empirical success in the literature [16].

The partial derivative of the second term in Equation 9 with respect to the parameter $\mathbf{w}^{l}$, for a training sample $\left(\mathbf{x}_{i}, y_{i}\right)$, is computed as

$$
\begin{aligned}
\frac{\partial \log p\left(y_{i} \mid \mathbf{x}_{i}^{l} ; \mathbf{w}^{l}\right)}{\partial \mathbf{w}^{l}} & =\sum_{\mathbf{h}^{l}} p\left(\mathbf{h}^{l} \mid y_{i}, \mathbf{x}_{i}^{l} ; \mathbf{w}^{l}\right) \frac{\partial F(\cdot)}{\partial \mathbf{w}^{l}} \\
& -\sum_{y^{\prime}, \mathbf{h}^{l}} p\left(y^{\prime}, \mathbf{h}^{l} \mid \mathbf{x}_{i}^{l} ; \mathbf{w}^{l}\right) \frac{\partial F(\cdot)}{\partial \mathbf{w}^{l}}
\end{aligned}
$$

Specific forms of the partial derivatives $\frac{\partial F(\cdot)}{\partial \mathbf{w}^{l}}$ with respect to $w_{y, h}^{l}$ and $w_{y, h, h}^{l}$ are the same as those in [16],
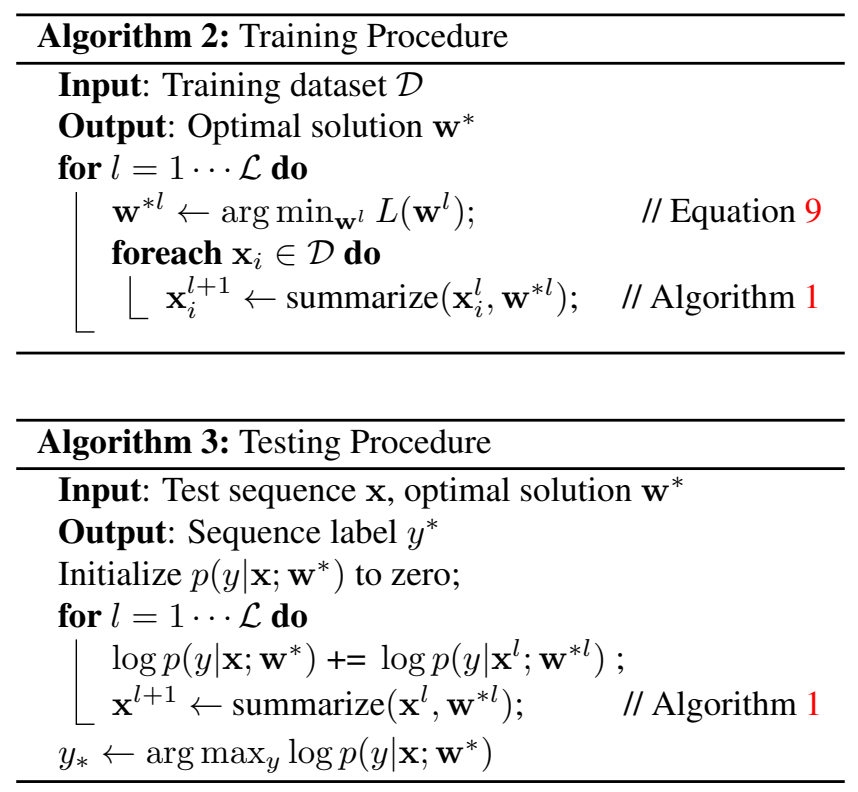

$\frac{\partial f^{2}(\cdot)}{\partial w_{y, h}^{l}}=\sum_{t} \mathbb{1}\left[y=y^{\prime}\right] \mathbb{1}\left[h_{t}^{l}=h^{\prime}\right]$ and $\frac{\partial f^{3}(\cdot)}{\partial w_{y, h, h}^{l}}=\sum_{t} \mathbb{1}[y=$ $\left.y^{\prime}\right] \mathbb{1}\left[h_{t}^{l}=h^{\prime}\right] \mathbb{1}\left[h_{t+1}^{l}=h^{\prime \prime}\right]$. For $w_{g, h}^{l}$ and $w_{g, d}^{l}$, they are $\frac{\partial f^{1}(\cdot)}{\partial w_{g, h}^{l}}=\sum_{t, g} \mathbb{1}\left[h_{t}^{l}=h^{\prime}\right] \psi_{g}\left(\mathbf{x}^{l}, t ; \mathbf{w}^{l}\right)$ and $\frac{\partial f^{1}(\cdot)}{\partial w_{g, d}^{l}}=$ $\sum_{t, g} w_{g, h}^{l} \frac{1}{\left|c\left(\mathbf{x}^{l}, t\right)\right|} \sum_{\mathbf{x}^{\prime}} g\left(\sum_{d} w_{g, d}^{l} x_{d}^{\prime}\right)\left(1-g\left(\sum_{d} w_{g, d}^{l} x_{d}^{\prime}\right)\right)$, respectively.

Training and Testing: Algorithm 2 and 3 show training and testing procedures, respectively. The training procedure involves, for each $l$, solving for $\mathrm{w}^{* l}$ and generating a sequence summary $\mathrm{x}^{l+1}$ for each sample in the dataset. The testing procedure involves adding up $\log p\left(y \mid \mathbf{x}^{l} ; \mathbf{w}^{* l}\right)$ computed from each layer and finding the optimal sequence label $y$ with the highest probability.

Note that if the summary produced the same sequence (i.e., $\mathbf{x}_{i}^{l+1}$ is equal to $\mathbf{x}_{i}^{l}$ ), we stop further grouping the sample $\mathbf{x}_{i}$, both in training and testing procedures. As a result, $\mathbf{x}^{l+1}$ is always shorter than $\mathbf{x}^{l}$.

Complexity Analysis: Because of this incremental optimization, the complexity grows only sublinearly with the number of layers considered. To see this, recall that solving an inference problem given a sequence takes $O\left(Y T H^{2}\right)$ and the sequence summarization takes $O(T \log T)$. With $\mathcal{L}$ layers considered, the complexity is $O\left(\mathcal{L} Y T H^{2}+\mathcal{L} T \log T\right)$; here, $T$ is a strictly decreasing function of the layer variable (because the length of $\mathrm{x}^{l+1}$ is always shorter than $\mathbf{x}^{l}$ ), and thus the complexity of our model increases sublinearly with the number of layers used.

\section{Experiments}

We evaluated the performance of our HSS model on three human activity datasets with different tasks, using different types of input features. 
ArmGesture [16]: The task in this dataset is to recognize various arm gestures based on upper body joint configuration. It contains 724 sequences from 6 action categories, with an average of 25 frames per sequence. Each frame is represented as a 20D feature vector: 2D joint angles and 3D coordinates for left/right shoulders and elbows.

Canal9 [25] $]^{2}$ : The task is to recognize agreement and disagreement during a political debate based on nonverbal audio-visual cues. It contains 145 sequences, with an average of 96 frames per sequence. Each frame is represented as a 10D feature vector: 2D prosodic features (F0 and energy) and 8D canonical body gestures, where the presence/absence of 8 gesture categories in each frame was manually annotated with binary values.

NATOPS [20] ${ }^{3}$ : The task is to recognize aircraft handling signals based on upper body joint configuration and hand shapes. It contains 2,400 sequences from 6 action categories, with an average of 44 frames per sequence. Each frame is represented as a 20D feature vector: 3D joint velocities for left/right elbows and wrists, and the probability estimates of four canonical hand gestures for each hand, encoded as $8 \mathrm{D}$ feature vector.

\subsection{Methodology}

We followed experimental protocols used in published work on each dataset: For the ArmGesture and Canal9 datasets we performed 5-fold cross-validation, for the NATOPS datasets we performed hold-out testing, using the samples from the first 5 subjects for testing, the second 5 subjects for validation, with the rest for training.

We varied the number of latent states $H \in\{4,8,12\}$ and the number of gate functions $G \in\{4,8,12\}$, and set the number of layers $\mathcal{L}=4$; for simplicity we set $H$ and $G$ to be the same across layers. The threshold constant in sequence summarization was varied $\tau \in\{0.1,0.5,1.0\}$ (see Algorithm 1). The $L_{2}$ regularization scale term $\sigma$ was varied $\sigma=\left\{10^{k} \mid k \in\{1,2,3\}\right\}$.

Since the objective function (Equation 9) is non-convex, we trained each model twice with different random initializations. The optimal configuration of all the hyperparameters we used were chosen based on the highest classification accuracy on the validation dataset.

\subsection{Results}

Table 1 and Table 2 shows experimental results on the ArmGesture and Canal9 datasets, respectively. We include previous results on each dataset reported in the literature; we also include the result obtained by us using CNF [15] with latent variables (HCNF). As can be seen, our approach

\footnotetext{
${ }^{2}$ The original dataset [25] contains over 43 hours of recording; to facilitate comparison with previous results we used the subset of the dataset [2]

${ }^{3}$ The original dataset [20] contains 9,600 sequences from 24 action categories; we used the subset of the dataset used in [21].
}

\begin{tabular}{|l|c|}
\hline Model & Mean Accuracy \\
\hline HMM (from [16]) & $84.83 \%$ \\
CRF (from [16]) & $86.03 \%$ \\
MM-HCRF (from [21]) & $93.79 \%$ \\
Quattoni et al. [16] & $93.81 \%$ \\
Shyr et al. [19] & $95.30 \%$ \\
Song et al. [21] & $97.65 \%$ \\
HCNF & $97.79 \%$ \\
\hline Our HSS Model & $\mathbf{9 9 . 5 9 \%}$ \\
\hline
\end{tabular}

Table 1. Experimental results from the ArmGesture dataset.

\begin{tabular}{|l|c|}
\hline Model & Mean Accuracy \\
\hline SVM (from [2]) & $51.89 \%$ \\
HMM (from [2]) & $52.29 \%$ \\
Bousmalis et al. [2] & $64.22 \%$ \\
Song et al. $[22]$ & $71.99 \%$ \\
HCNF & $73.35 \%$ \\
\hline Our HSS Model & $\mathbf{7 5 . 5 6 \%}$ \\
\hline
\end{tabular}

Table 2. Experimental results from the Canal9 dataset.

outperforms all the state-of-the-art results on the ArmGesture and Canal9 datasets. Notably, our approach achieves a near-perfect accuracy on the ArmGesture dataset (99.59\%).

For the NATOPS dataset, the state-of-the-art result is $87.00 \%$ by Song et al. [21]. Their approach used a multiview HCRF to jointly learn view-shared and view-specific hidden dynamics, where the two views are defined as upper body joint configuration and hand shape information. Even without considering the multi-view nature of the dataset (we perform an early-fusion of the two views), our approach achieved a comparable accuracy of $85.00 \%$. This is still a significant improvement over various previous results using an early-fusion: HMM (from [21], 76.67\%), HCRF (from [21], 76.00\%), and HCNF (78.33\%).

\subsection{Detailed Analysis and Discussions}

For detailed analysis we evaluated whether our hierarchical representation is indeed advantageous over a single representation, and how our sequence summarization in the latent space differs from the other approaches.

1) Hierarchical vs. single optimal representation: While our results show significant improvements over previous sequence learning models, they do not prove the advantage of learning from hierarchical sequence summary representation, as opposed to learning from only the optimal layer inside the hierarchy (if any). To this end, we compared our approach to the single (top) layer approach by computing during the testing procedure $p(y \mid \mathbf{x} ; \mathbf{w})=p\left(y \mid \mathbf{x}^{\mathcal{L}} ; \mathbf{w}^{\mathcal{L}}\right)$, varying $\mathcal{L}=\{2,3,4\}$; the training procedure was the same as Algorithm 2 (otherwise the obtained sequence summary is not optimal). 
(a) ArmGesture

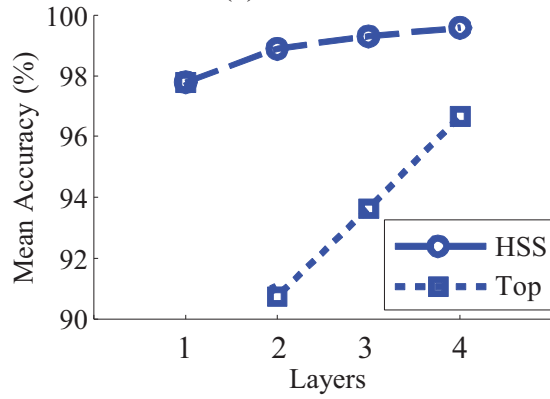

(d) ArmGesture

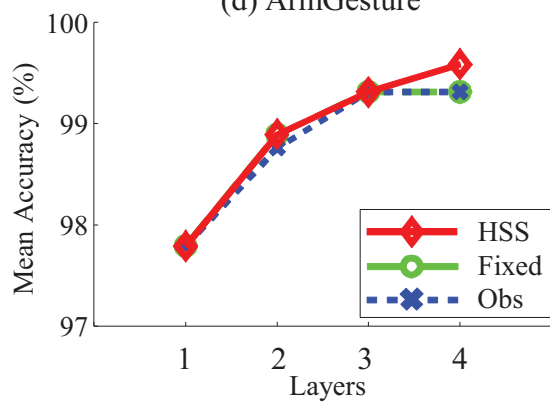

(b) Canal9

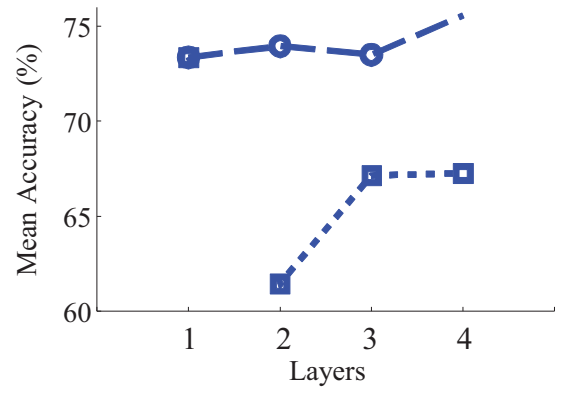

(e) Canal9

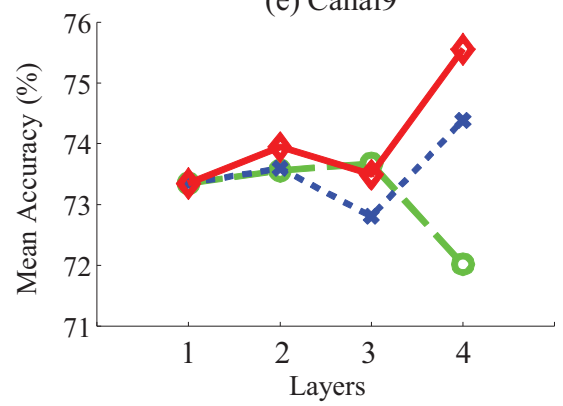

(c) NATOPS

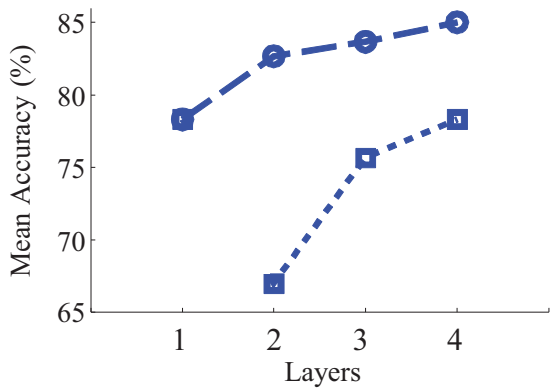

(f) NATOPS

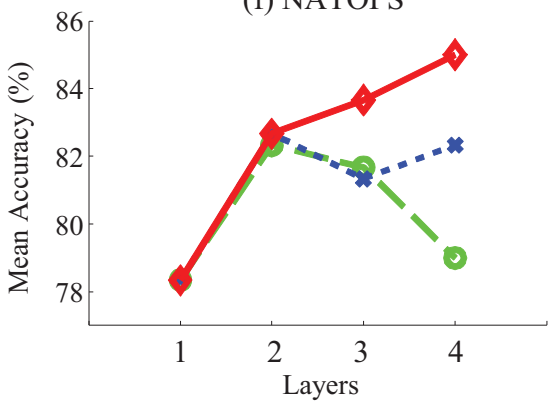

Figure 4. Detailed analysis results. The top row (a)-(c) shows experimental results comparing hierarchical (HSS) and single optimal (top) representation approaches, the bottom row (d)-(f) shows the results on three different sequence summarization approaches.

Figures 4 (a)-(c) show the mean classification accuracy as a function of $\mathcal{L}$, the number of layers, on all three datasets. Our "HSS" approach always outperformed the "Top" approach. Paired t-tests showed that the differences were statistically significant in all three datasets $(p<.001)$. This shows that there is no single representation that is as discriminative as the hierarchical representation.

2) Different sequence summarization algorithms: Our sequence summarization produces groups of temporally neighboring observations that have similar semantic meaning in the latent space. We compare this to two different approaches: One approach simply collapses every $l$ consecutive observations and obtain a sequence of length $T / l$ at each layer $l$ ("Fixed" in Figure 4). Another approach produces groups of observations that are similar in the feature space, with a similarity metric defined as $w_{s t}=\left|\mathbf{x}_{s}-\mathbf{x}_{t}\right|$ and with the threshold range $\tau=\{1,5,10\}$ ("Obs" in Figure 4).

As can be seen in Figures 4 (d)-(f), our approach outperforms the two other approaches on the Canal9 and NATOPS datasets; on the ArmGesture dataset, performance saturates towards near perfect accuracy. The Fixed approach collapses observations as long as there is more than one, even if they contain discriminative information individually, which may cause over-grouping. Our result supports this hypothesis, showing that the performance started to decrease after $\mathcal{L}>3$ on the Canal9 and NATOPS datasets.
The Obs approach groups observations using input features, not the corresponding posteriors $p(\mathbf{h} \mid y, \mathbf{x} ; \mathbf{w})$ in the latent space. There are a number of difficulties when dealing with input features directly, e.g., different scales, range of values, etc, which makes the approach sensitive to the selected feature space. Our approach, on the other hand, uses latent variables that are defined in the scale [0:1] and contains discriminative information learned via mathematical optimization. We can therefore expect that, as can be seen in our results, our approach is more robust to the selection of the scale/range as well as the threshold parameter $\tau$, resulting in overall better performance.

\section{Conclusion}

We presented a hierarchical sequence summarization (HSS) model for action recognition, and showed that it achieves the best published results on the ArmGesture and Canal9 datasets. We showed how learning from a hierarchical representations is important, and how grouping observations that are similar in the latent space has several advantages over other methods.

Our model is quite general and can work with different types of input features. By being feature agnostic, our model is applicable to other domains dealing with temporal sequence data, such as multimodal social signal processing. We plan to test our model on these and other real-world sequence analysis tasks. 


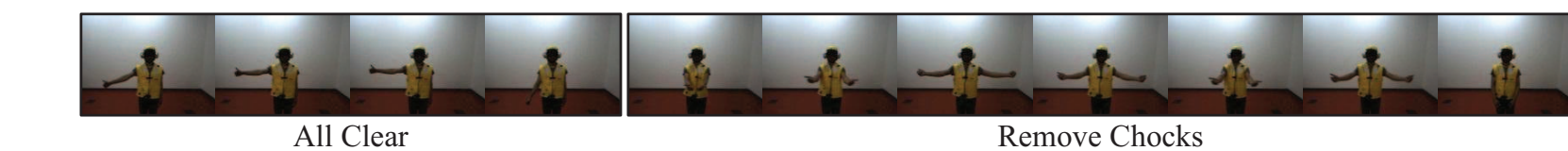

All Clear

Remove Chocks

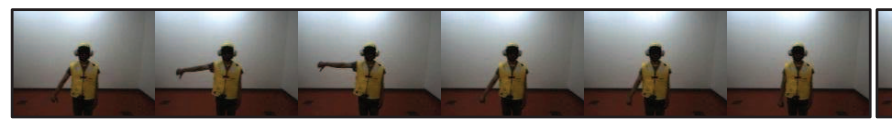

Not Clear

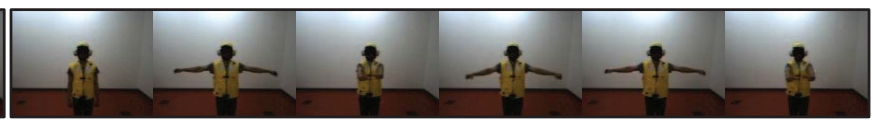

Insert Chocks

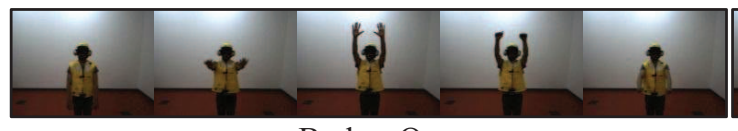

Brakes On

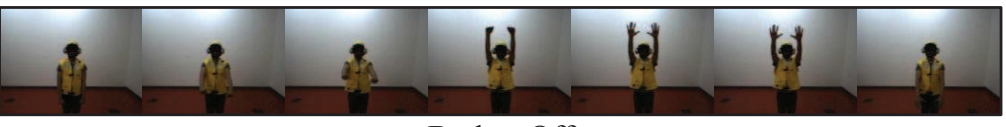

Brakes Off

Figure 5. Inferred sequence summaries on the NATOPS dataset [20]. Each super observation represents key transitions of each action class. For the purpose of visualization we selected the middle frame from each super observation at the 4-th layer.

\section{References}

[1] Y. Bengio. Learning deep architectures for AI. FTML, 2(1), 2009. 2, 3, 4

[2] K. Bousmalis, L.-P. Morency, and M. Pantic. Modeling hidden dynamics of multimodal cues for spontaneous agreement and disagreement recognition. In $F G, 2011.6$

[3] P. F. Felzenszwalb and D. P. Huttenlocher. Efficient graphbased image segmentation. IJCV, 59(2), 2004. 2, 4

[4] J. R. K. Hartline. Incremental Optimization. PhD thesis, Cornell University, 2008. 5

[5] G. E. Hinton, S. Osindero, and Y. W. Teh. A fast learning algorithm for deep belief nets. NECO, 18(7), 2006. 2

[6] G. B. Huang, H. Lee, and E. G. Learned-Miller. Learning hierarchical representations for face verification with convolutional deep belief networks. In CVPR, 2012. 2

[7] P. Kohli, L. Ladicky, and P. H. S. Torr. Robust higher order potentials for enforcing label consistency. IJCV, 82, 2009. 1

[8] A. Kovashka and K. Grauman. Learning a hierarchy of discriminative space-time neighborhood features for human action recognition. In $C V P R, 2010.2$

[9] I. Laptev. On space-time interest points. IJCV, 64(2-3), 2005. 2

[10] I. Laptev, M. Marszalek, C. Schmid, and B. Rozenfeld. Learning realistic human actions from movies. In $C V P R$, 2008. 2, 3

[11] S. Lazebnik, C. Schmid, and J. Ponce. Beyond bags of features: Spatial pyramid matching for recognizing natural scene categories. In $C V P R, 2006.1$

[12] Q. V. Le, W. Y. Zou, S. Y. Yeung, and A. Y. Ng. Learning hierarchical invariant spatio-temporal features for action recognition with independent subspace analysis. In $C V P R$, 2011. 1, 2, 4

[13] J. C. Niebles and L. Fei-Fei. A hierarchical model of shape and appearance for human action classification. In $C V P R$, 2007. 2

[14] J. Nocedal and S. J. Wright. Numerical Optimization. Springer-Verlag, 1999. 5

[15] J. Peng, L. Bo, and J. Xu. Conditional neural fields. In NIPS, 2009. 2, 6
[16] A. Quattoni, S. B. Wang, L.-P. Morency, M. Collins, and T. Darrell. Hidden conditional random fields. PAMI., 29(10), 2007. 2, 3, 5, 6

[17] M. Ranzato, J. Susskind, V. Mnih, and G. E. Hinton. On deep generative models with applications to recognition. In CVPR, 2011. 1

[18] R. Salakhutdinov and G. E. Hinton. An efficient learning procedure for deep boltzmann machines. NECO, 24(8), 2012. 2

[19] A. Shyr, R. Urtasun, and M. I. Jordan. Sufficient dimension reduction for visual sequence classification. In CVPR, 2010. 6

[20] Y. Song, D. Demirdjian, and R. Davis. Tracking body and hands for gesture recognition: Natops aircraft handling signals database. In $F G, 2011.6,8$

[21] Y. Song, L.-P. Morency, and R. Davis. Multi-view latent variable discriminative models for action recognition. In $C V P R$, 2012. 6

[22] Y. Song, L.-P. Morency, and R. Davis. Multimodal human behavior analysis: learning correlation and interaction across modalities. In ICMI, 2012. 6

[23] J. Sun, X. Wu, S. Yan, L. F. Cheong, T.-S. Chua, and J. Li. Hierarchical spatio-temporal context modeling for action recognition. In $C V P R, 2009.2$

[24] K. Tang, F.-F. Li, and D. Koller. Learning latent temporal structure for complex event detection. In $C V P R, 2012.2$

[25] A. Vinciarelli, A. Dielmann, S. Favre, and H. Salamin. Canal9: A database of political debates for analysis of social interactions. In ACII, 2009. 6

[26] J. Wang, Z. Chen, and Y. Wu. Action recognition with multiscale spatio-temporal contexts. In CVPR, 2011. 2

[27] Y. Wang and G. Mori. Hidden part models for human action recognition: Probabilistic versus max margin. PAMI, 33(7), 2011. 2

[28] D. Yu and L. Deng. Deep-structured hidden conditional random fields for phonetic recognition. In INTERSPEECH, 2010. 2 